\title{
Visceral leishmaniasis in a six-year-old patient - unexpected diagnosis: case study
}

\author{
Katarzyna Sierżęga-Staykov, Jadwiga Węcławek-Tompol, Ewa Kawecka, Halina Pela, \\ Bernarda Kazanowska \\ Department of Paediatric Bone Marrow Transplantation, Oncology, and Haematology, Wroclaw Medical University, \\ Wroclaw, Poland
}

\section{ABSTRACT}

Visceral leishmaniasis is a parasitic disease caused by protozoa of the Leishmania genus and transmitted by the bites of phlebotomine sandfly species. The disease may be a diagnostic challenge in nonendemic countries. The following study presents a case of a six-year-old girl with recurrent fever, hepatosplenomegaly, lymphadenopathy, and pancytopaenia. During the diagnostic work-up acute leukaemia, metabolic diseases, and connective tissue diseases were excluded. A second examination of the bone marrow revealed macrophages containing inclusions typical of Leishmania amastigotes. The diagnosis was confirmed by identification of the parasite's DNA in a PCR test of a bone marrow sample and serologic detection of antibodies to Leishmania spp. Treatment with liposomal amphotericin B was administered with good effect.

\section{KEY WORDS:}

visceral leishmaniasis, parasitic disease, child.

\section{INTRODUCTION}

The leishmaniases are a group of parasitic diseases caused by protozoa of the Leishmania genus and transmitted by the bites of phlebotomine sandfly species. Visceral leishmaniasis (VL), also known as kala-azar, is the most severe form of leishmaniasis. Approximately 50,000 to 90,000 new cases of VL are detected worldwide annually. The major risk factors for VL are malnutrition, poor housing, human migration, and environmental and climate changes. Although there are 75 endemic countries for VL, about $90 \%$ of all global cases are limited to the following seven: Brazil, Ethiopia, India, Kenya, Somalia, South Sudan, and Sudan [1,2]. This potentially fatal systemic infection is mainly characterised by irregular fever and splenomegaly. Nevertheless, affected patients may present an array of other symptoms including weight loss, hepatomegaly, pancytopaenia, and lymphadenopathy. Hypergammaglobulinaemia is common; however, the presence of autoimmune antibodies may confuse the presentation, especially in travellers or migrants [3]. Febrile splenomegaly occurs in many infectious diseases; therefore, a suspicion of VL needs to be confirmed by Leishmania-specific laboratory tests. The classical test is based on microscopic observation of the amastigote form of the parasite in tissue aspirates. The specificity of the method is high, but the sensitivity of microscopy varies between different tissues and is highest for spleen and bone marrow aspirates [4]. Serological tests based on the detection of antibodies to Leishmania from patients' serum are currently the gold standard and the most widely used method for control of VL in endemic areas $[5,6]$. The major limitation of serological diagnosis is the persistence of the antibodies for several years after cure and

\section{ADDRESS FOR CORRESPONDENCE:}

Katarzyna Sierżęga-Staykov, Department of Paediatric Bone Marrow Transplantation, Oncology, and Haematology,

Wroclaw Medical University, 213 Borowska St., 50-556 Wroclaw, Poland, ORCID: 0000-0003-4057-1062,

e-mail: katarzyna.sierzega@yahoo.com 
therefore the inability to reliably control relapse or distinguish between current, asymptomatic, or past infections $[4,5]$. The detection of parasite DNA by polymerase chain reaction $(\mathrm{PCR})$ in aspirates of blood or bone marrow is the most sensitive and specific method, but it is restricted to research centres $[4,6]$. The treatment of VL is based on chemotherapy. The primary drugs are pentavalent antimonials, but clinical resistance and treatment failure is becoming more pronounced. Alternative medications such as miltefosine, paromomycin, and amphotericin B are increasingly used worldwide [7]. Furthermore, the development of a vaccine that prevents Leishmania infection, halts its progression, or limits transmission are research priorities. Currently, only three vaccines are being tested in clinical trials [3], and several in are in preclinical development [8].

\section{CASE REPORT}

The case report refers to a six-year-old girl who was admitted to a paediatric oncology clinic with a suspicion of acute leukaemia. Symptoms had started five months prior to admission when mild anaemia and thrombocytopaenia were found in control blood count. The patient was treated with iron therapy, folic acid, and vitamin B6 by the primary care physician. Over the following months subsequent symptoms appeared - malaise, lower extremity pain, epistaxis, bruising tendency, abdominal distension, recurrent fever, and pancytopaenia. The child was referred to the clinic for further diagnostics. On admission, the physical examination revealed pallor, caput medusae, tachycardia, hepatosplenomegaly, and cervical lymphadenopathy. Laboratory tests confirmed pancytopaenia $\left(\right.$ WBC $3.79 \times 10^{9} / \mathrm{l}$, RBC $3.34 \times 10^{12} / \mathrm{l}$, Hb $7.3 \mathrm{~g} / \mathrm{dl}$, PLT $\left.53 \times 10^{9} / 1\right)$, lymphocytosis on peripheral blood smear, and increased values of inflammatory markers, liver enzymes, and D-dimer levels. Hypergammaglobulinaemia and elevated levels of autoimmune antibodies were also detected. Abdominal computed tomography (CT) showed massive splenomegaly, moderate hepatomegaly, and multiple, polycyclic structures along the celiac trunk, which might suggested pathological lymph nodes (Fig. 1). Thoracic CT revealed mediastinal and axillary lymphadenopathy. On the basis of bone marrow examination acute leukaemia was excluded. The patient was consulted with a rheumatologist; after negative tests of double-stranded DNA, anticardiolipin, and lupus anticoagulant, differential diagnosis of systemic lupus erythematosus was excluded. Subsequently, the girl was referred to the Department of Metabolic Diseases, where inherited metabolic disorders were ruled out, including the most probable - Gaucher disease. Despite the use of broad-spectrum antibiotics, and antiviral and antifungal drugs, irregular fever persisted and the patient required regular transfusions of blood. At that time, re-examination of bone marrow was carried out and it showed inclusions in macrophages typical of amastigote forms of Leishmania (Fig. 2). The diagnosis was confirmed by identification of the parasite's DNA by PCR and serologic detection of antibodies to Leishmania spp. The infection was probably acquired two years earlier during holidays in Cro-
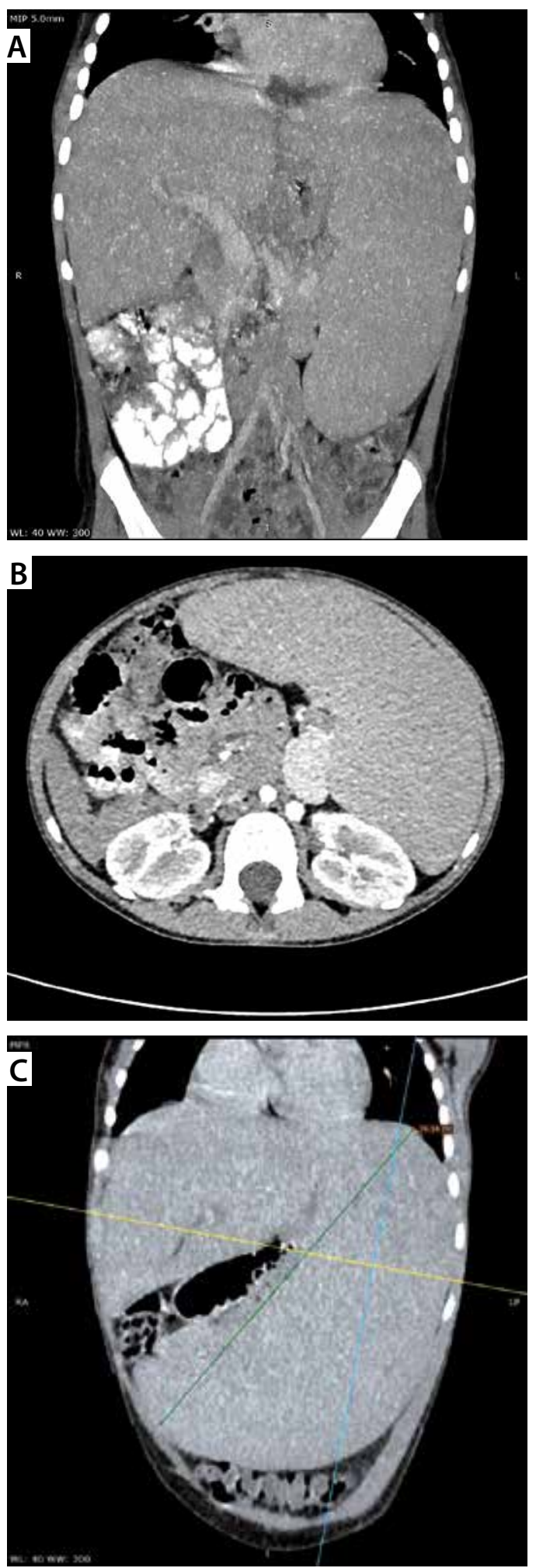

FIGURE 1. Abdominal CT of the patient showing: A) massive hepatosplenomegaly, B) enlargement of the spleen visualised on the axial projection, and $\mathrm{C}$ ) and enlargement of the spleen visualised with bipolar splenic diameter 

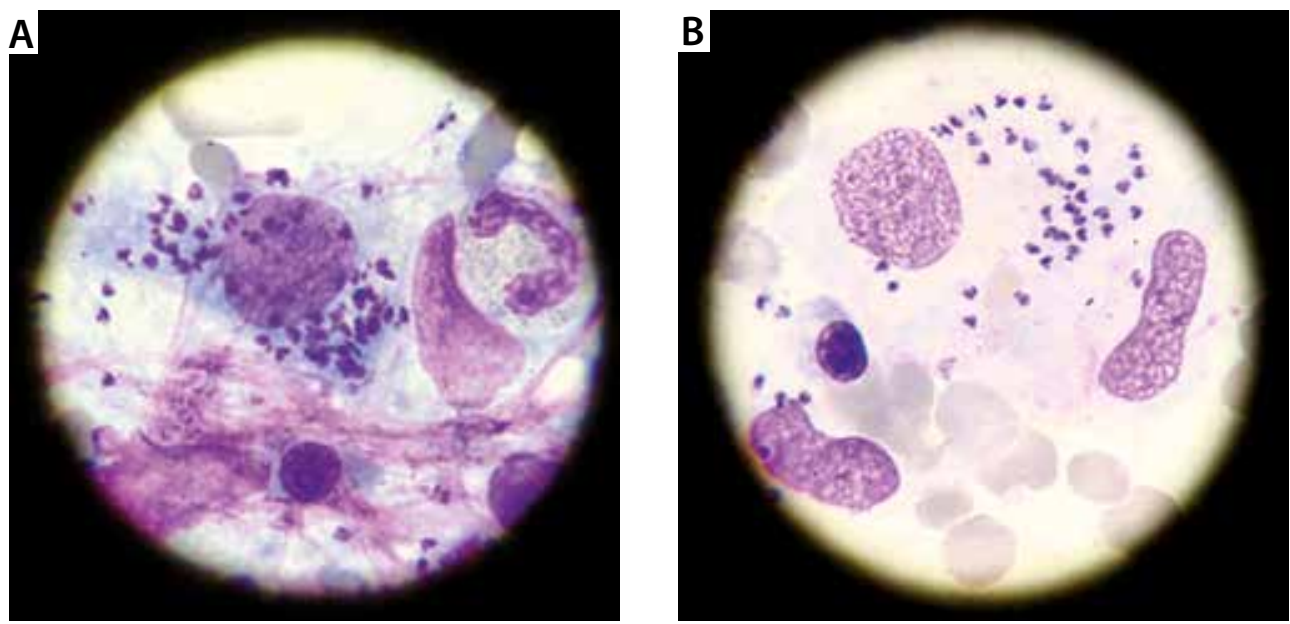

FIGURE 2. Microscopy findings of amastigotes of Leishmania in macrophages of the patient ( $A$ and $B$ )

atia and Bosnia and Herzegovina, which are endemic regions for VL. Therapy of liposomal amphotericin B was administered (at a dose of $3 \mathrm{mg}$ per $\mathrm{kg}$, for 10 days) [9-12]. We observed good parasitological response to the treatment: improvement of the patient's overall condition, reduction of the size of the spleen, and normalisation of the peripheral blood cell count [12].

\section{DISCUSSION}

Due to the increasing number of people undertaking long-distance travel, the incidence of cases of endemic diseases imported to nonendemic countries is on the rise [13]. The diagnosis of VL in nonendemic areas, including Poland, may be challenging, although the clinical presentation is usually similar to that in endemic areas. This is mainly due to the fact that the clinical possibility of VL in the differential diagnosis is very low in patients from nonendemic regions $[14,15]$. Delay in diagnosis and treatment may lead to serious complications and even a fatal outcome of VL [16]. European endemic countries are located mainly in the Mediterranean basin. Nonetheless, leishmaniasis is still considered as a tropical disease by many European physicians and public health experts [17]. It is noteworthy that the data on cases of the disease in children are limited, so the discussion is difficult. Chandra et al. analysed 27 cases of VL in nonendemic regions [18]. The study showed that fever and hepatosplenomegaly were the most common clinical presentations of VL. According to haematological findings, all patients were anaemic, and pancytopaenia was observed in $96.2 \%$. The diagnosis of VL was not initially suspected in $81.4 \%$ of the cases. The study indicated that the substantial delay between the onset of symptoms and the final diagnosis is mainly caused by its presence in a nonendemic region and overlapping clinical features of other conditions typical of this area. Interestingly, several European studies [19-21] showed that very few of the imported infections have been acquired in countries that account for over $90 \%$ of VL cases worldwide (Brazil, Ethiopia, India, Ken- ya, Somalia, South Sudan, and Sudan). The recorded data emphasise the differences in the epidemiology of VL in travellers and inhabitants of epidemic regions. Most of the patients with travel-acquired VL were successfully treated with liposomal amphotericin B [19-21]. To date, no vaccines or preventive drugs are available, so personal protective measures are recommended to travellers to minimise exposure to sandfly bites with the following: protective clothing, insect repellent to exposed skin, and minimising nocturnal outdoor activities $[9,10]$.

\section{CONCLUSIONS}

Visceral leishmaniasis is a rare cause of prolonged, irregular fever, hepatosplenomegaly, and pancytopaenia in children in nonendemic regions. The clinical manifestation of VL may mimic symptoms of other paediatric conditions, including oncological, metabolic, and connective tissue diseases. This case report reinforces the need for consideration of VL in the differential diagnosis whenever the symptoms occur, even in nonendemic areas.

\section{DISCLOSURE}

The authors declare no conflict of interest.

\section{REFERENCES}

1. World Health Organization Global Health Observatory, Leishmaniasis (2017). http://www.who.int/gho/neglected_diseases/leishmaniasis/en/.

2. World Health Organization Media Centre, Leishmaniasis (2018). http://www.who.int/mediacentre/factsheets/fs375/en/.

3. Burza S, Croft SL, Boelaert M. Leishmaniasis. Lancet 2018; 392 : 951-970.

4. WHO. Control of the leishmaniases. World Health Organization, Geneva 2010.

5. Rijal S, Chappuis F, Boelaert M. Challenges in the Diagnosis of Visceral Leishmaniasis on the Indian Subcontinent. In: Kala Azar in South Asia. Current Status and Challenges Ahead, Jha T-K, Noiri E (eds.). Springer, Dordrecht 2011; 59-67. 
6. Srividya G, Kulshrestha A, Singh R, et al. Diagnosis of visceral leishmaniasis: developments over the last decade. Parasitol Res 2012; 110: 1065-1078.

7. Ponte-Sucre A, Gamarro F, Dujardin J-C, et al. Drug resistance and treatment failure in leishmaniasis: A 21st century challenge. PLoS Negl Trop Dis 2017; 11: e0006052.

8. Kumar R, Engwerda C. Vaccines to prevent leishmaniasis. Clin Transl Immunology 2014; 3, e13.

9. Centers for Disease Control and Prevention, Parasites - Leishmaniasis, Resources for Health Professionals (2017). https://www.cdc. gov/parasites/leishmaniasis/health_professionals/index.html\#tx

10. Sundar S, Agarwal D. Visceral Leishmaniasis-Optimum Treatment Options in Children. Pediatr Infect Dis J 2018; 37: 492-494.

11. Louis DS, Bern C, Adler-Moore J, et al. Liposomal Amphotericin B for the Treatment of Visceral Leishmaniasis. Clin Infect Dis 2006; 43: 917-924.

12. Aronson N, Herwaldt BL, Libman M, et al. Diagnosis and Treatment of Leishmaniasis: Clinical Practice Guidelines by the Infectious Diseases Society of America (IDSA) and the American Society of Tropical Medicine and Hygiene (ASTMH). Clin Infect Dis 2016; 63: e202-e264.

13. Górski S, Wiercińska-Drapało A. Visceral leishmaniasis as a threat for non-endemic countries. Wiad Parazytol 2009; 55: 195-200.

14. Raina RK, Raina S, Sharma M. Visceral leishmaniasis-associated hemophagocytosis: A tale of two unexpected diagnoses from a nonendemic region. Trop Parasitol 2017; 7: 56-58.

15. Raina S, Mahesh DM, Kaul R, et al. A new focus of visceral leishmaniasis in the Himalayas, India. J Vector Borne Dis 2009; 46: 303-306.

16. Mathur SB, Arya AK. Nonmigrant children with visceral leishmaniasis from the nonendemic area of Uttarakhand. J Trop Pediatr 2014; 60: 322-325.

17. Gradoni L. Epidemiological surveillance of leishmaniasis in the European Union: operational and research challenges. Euro Surveill 2013; 18: pii=20539.

18. Chandra H, Chandra S, Kaushik RM. Visceral Leishmaniasis with Associated Common, Uncommon, and Atypical Morphological Features on Bone Marrow Aspirate Cytology in Nonendemic Region. J Trop Med 2013; 2013: 861032.

19. Weitzel T, Mühlberger N, Jelinek T, et al. Imported leishmaniasis in Germany 2001-2004: data of the SIMPID surveillance network. Eur J Clin Microbiol Infect Dis 2005; 24: 471-476.

20. Malik AN, John L, Bruceson AD, et al. Changing pattern of visceral leishmaniasis, United Kingdom, 1985-2004. Emerg Infect Dis 2006; 12: 1257-1259.

21. Harms G, Schonian G, Feldmeier H. Leishmaniasis in Germany. Emerg Infect Dis 2003; 9: 872-875. 